\title{
Regressividade financeira: investigação do impacto econômico durante a pandemia do coronavírus COVID-19 sobre a família brasileira
}

\author{
Financial regressivity: investigating the economic impact during the COVID-19 coronavirus \\ pandemic on the brazilian family \\ Regresividad financiera: investigacón del impacto económico durante la pandémica COVID-19 \\ coronavírus en la familia brasileña
}

Recebido: 12/08/2021 | Revisado: 18/08/2021 | Aceito: 20/08/2021 | Publicado: 22/08/2021

\author{
Lizandro da Conceição Costa Melonio \\ ORCID: https://orcid.org/0000-0002-7007-7770 \\ Faculdade Futura, Brasil \\ E-mail: lizandro.ccmelonio@gmail.com \\ Márcia Lima Santos de Cezere \\ ORCID: https://orcid.org/0000-0002-1118-3784 \\ E-mail: marciacezere@ifsul.edu.br \\ Wemerson de Castro Oliveira \\ ORCID: https://orcid.org/0000-0001-7256-265X \\ E-mail: wemersonoliveira@ifsul.edu.br
} Instituto Federal de Educação, Ciência e Tecnologia Sul-rio-grandense, Brasil Instituto Federal de Educação, Ciência e Tecnologia Sul-rio-grandense, Brasil

\begin{abstract}
Resumo
Objetivou-se com essa pesquisa investigar o impacto econômico no início da pandemia do coronavírus COVID-19 sobre a família brasileira. O estudo caracteriza-se como quantitativo e qualitativo descritivo e/ou exploratório. Além disso, possui um delineamento de pesquisa de campo, utilizando como instrumento de coleta de dados um questionário pré-estruturado com 19 perguntas objetivas, divididas nas duas categorias: (a) perfil ou dados pessoais; e (b) o impacto financeiro causado pela pandemia do COVID-19. Utilizou-se o método de escores para definir a tendência de endividamento dos indivíduos. A partir da unidade amostral ( $n=336$ respondentes) ficou evidente o despreparo financeiro da grande maioria dos entrevistados para o enfrentamento da pandemia. No entanto, um considerado percentual possui reservas financeiras para um período emergencial. Já no início da pandemia, grande parte das famílias dos entrevistados tiveram sua renda mensal reduzida sendo necessária a realização de cortes para manter o controle financeiro. A partir disso, as despesas priorizadas no período de pandemia seriam alimentação e saúde. Com apenas dois meses de início do isolamento no Brasil, um percentual de 13,7 dos entrevistados haviam contraído dívidas pelas necessidades que surgiram decorrentes da pandemia: $73,5 \%$ e $26,5 \%$ destes estão abaixo e acima do escore médio de endividamento, respectivamente. Curiosamente, $24,1 \%$ dos respondentes possuem dúvidas e 4,8\% não pretendem economizar recursos para eventuais contingências após esse momento pandêmico. De maneira conclusiva, as famílias brasileiras não estão preparadas financeiramente para o enfrentamento de situações emergenciais como de uma pandemia e que esse despreparo está associado à falta da educação financeira.
\end{abstract}

Palavras-chave: Crise financeira; Educação financeira; Gestão financeira; COVID-19.

\begin{abstract}
The objective of this research was to investigate the economic impact on the beginning of the COVID-19 coronavirus pandemic on the Brazilian family. The study is characterized as quantitative and qualitative descriptive and/or exploratory. In addition, it has a field research design, using as a data collection instrument a pre-structured questionnaire with 19 objective questions, divided into two categories: (a) profile or personal data; and (b) the financial impact caused by the COVID-19 pandemic. The scoring method was used to define the indebtedness trend of individuals. From the sampling unit ( $n=336$ respondents), the financial unpreparedness of the vast majority of respondents to face the pandemic was evident. However, a considered percentage has financial reserves for an emergency period. At the beginning of the pandemic, most of the interviewees' families had their monthly income reduced, making cuts necessary to maintain financial control. From then on, the expenses prioritized during the pandemic period would be food and health. With only two months of the beginning of isolation in Brazil, a percentage of 13.7 of respondents had incurred debt due to the needs that arose from the pandemic: $73.5 \%$ and $26.5 \%$ of these are below and above the average debt score, respectively. Interestingly, $24.1 \%$ of respondents have doubts and $4.8 \%$ do
\end{abstract}


not intend to save resources for possible contingencies after this pandemic moment. Conclusively, Brazilian families are not financially prepared to face emergency situations such as a pandemic and that this lack of preparation is associated with a lack of financial education.

Keywords: Financial crisis; Financial education; Financial management; COVID-19.

\section{Resumen}

El objetivo de esta investigación fue investigar el impacto económico del inicio de la pandemia del coronavirus COVID-19 en la familia brasileña. El estudio se caracteriza por ser descriptivo y / o exploratorio cuantitativo y cualitativo. Además, cuenta con un diseño de investigación de campo, utilizando como instrumento de recolección de datos un cuestionario preestructurado con 19 preguntas objetivas, divididas en dos categorías: (a) perfil o datos personales; y (b) el impacto financiero causado por la pandemia de COVID-19. El método de puntuación se utilizó para definir la tendencia de endeudamiento de los individuos. A partir de la unidad de muestreo $(\mathrm{n}=336$ encuestados) fue evidente la falta de preparación financiera de la gran mayoría de los encuestados para hacer frente a la pandemia. Sin embargo, un porcentaje considerado tiene reservas financieras para un período de emergencia. Al comienzo de la pandemia, a la mayoría de las familias de los entrevistados se les redujo el ingreso mensual, lo que hizo necesarios recortes para mantener el control financiero. A partir de ese momento, los gastos priorizados durante el período pandémico serían los de alimentación y salud. Con solo dos meses del inicio del aislamiento en Brasil, un porcentaje del 13,7\% de los encuestados había incurrido en deudas debido a las necesidades surgidas de la pandemia: el 73,5\% y el $26,5 \%$ de estos están por debajo y por encima del puntaje promedio de deuda, respectivamente. Curiosamente, el $24,1 \%$ de los encuestados tiene dudas y el $4,8 \%$ no pretende ahorrar recursos para posibles contingencias tras este momento pandémico. En conclusión, las familias brasileñas no están preparadas financieramente para enfrentar situaciones de emergencia como una pandemia y que esta falta de preparación se asocia con la falta de educación financiera.

Palabras clave: Crisis financiera; Educación financiera; Gestión financeira; COVID-19.

\section{Introdução}

Atualmente, estamos vivendo no mundo uma pandemia, instituída pela Organização Mundial da Saúde (OMS), em 11 de março de 2020, provocada por um tipo de vírus classificado na família dos vírus tipo corona, ou coronavírus. Esta doença chamada de covid-19 é causada pelo coronavírus SARS-CoV-2, e pode levar a um quadro clínico crítico e até mesmo ao óbito (BRASIL, 2020). Segundo a Universidade \& Medicine John Hopkins, até o dia 12 de agosto de 2021, pelo menos 205.264.685 casos da doença foram confirmados em mais de 210 países e territórios, com grandes surtos nos Estados Unidos, Índia, Indonésia, Brasil, Reino Unido, Irã e Rússia (UNIVERSITY \& MEDICINE JOHN HOPKINS, 2021). Após a OMS ter declarado Emergência em Saúde Pública de Importância Internacional, foi instituído no Brasil, por meio da portaria n 188/2020 no dia 30 de janeiro, a Emergência em Saúde Pública. Ainda como medidas para combater a crise sanitária, o governo federal editou e o Congresso Nacional aprovou a Lei $\mathrm{n}^{\circ} 13.979 / 2020$, que traz as medidas de enfrentamento à emergência e seus efeitos. Já com o objetivo de combater a crise financeira decorrente, foi definido o estado de calamidade pública através do Decreto Legislativo n 6/2020 (Verdélio, 2020).

Globalmente, a crise causada pela pandemia do COVID-19, já está instalada e seu impacto econômico é mais grave que as pandemias causadas pelo SARS ou MERS, segundo Scott (2020). Para Baldwin (2020), diferentemente de outras crises recentes, a gerada pela pandemia do COVID-19 instituiu três tipos de choques econômicos: o primeiro, atinge o setor produtivo através do afastamento dos trabalhadores doentes, tendo como consequência o desemprego temporário; o segundo, está relacionado com as contenções que foram geradas devido ao isolamento, fechamento de fábricas e estabelecimentos comerciais e proibições de viagens; e o terceiro, se relaciona com o choque de expectativas em relação a economia futura. O autor ainda ressalta que esses choques atrapalham o fluxo de dinheiro, devido às dificuldades financeiras das famílias; choque nas importações; ruptura nas cadeias de suprimentos internacionais e domésticas; falência forçada das empresas; e a interrupção do trabalho por causa das demissões e afastamento para o isolamento.

Além disso, o impacto direto, relacionados a saúde e vidas perdidas, e o impacto indireto, resultante do distanciamento social e fechamento obrigatório de negócios, geram um alto custo, segundo Mulligan, Murphy e Topel (2020). Os autores, 
ainda estimam que o custo para retardar a pandemia, através de um desligamento nacional, de atividades econômicas não essenciais seriam de 7 trilhões de dólares por ano, aproximadamente 20 bilhões de dólares por dia.

Diante deste contexto, há a necessidade de buscar ou resgatar a educação financeira, além de despertar um interesse pelo olhar do contágio financeiro (Forbes \& Rigobon, 2001; Seth \& Panda, 2018). Com isso, a Educação Financeira pode ser entendida como "um processo de concepção de conhecimento que pode melhorar a capacidade financeira das pessoas, para que possam tomar decisões sábias e seguras, se integrar mais à sociedade e usufruir da busca ativa da realização pessoal" (Amadeu, 2009). Ademais, Cordeiro e colaboradores (2018) entendem que a "Educação Financeira é um processo educativo que, por meio de aplicação de métodos próprios, desenvolve atividades para auxiliar os consumidores a orçar e gerir a sua renda, a poupar e a investir [...]" e Gallery e colaboradores (2011, p. 288), que a "Educação Financeira é a capacidade de fazer julgamentos inteligentes e decisões eficazes em relação ao uso e gestão do dinheiro".

Já para a Organização de Cooperação e de Desenvolvimento Econômico - OCDE (2005):

"Educação Financeira é o processo mediante o qual os indivíduos e as sociedades melhoram a sua compreensão em relação aos conceitos e produtos financeiros, de maneira que, com informação, formação e orientação, possam desenvolver os valores e as competências necessários para se tornarem mais conscientes das oportunidades e riscos neles envolvidos e, então, poderem fazer escolhas bem informadas, saber onde procurar ajuda e adotar outras ações que melhorem o seu bem-estar financeiro" (OCDE, 2005, p. 26).

Com isso, devemos considerar que a Educação Financeira proporciona aos indivíduos os conhecimentos e informações necessários para que possam se adaptar às suas condições econômicas de forma micro e pessoal (financeira própria) e macro, levando em consideração as condições do país e do mundo que podem afetar sua vida pessoal, familiar e empresarial (Ross et al., 2009). Para Kruger (2014) não é suficiente apenas entender a educação financeira, precisa também saber aplicar de forma saudável, até mesmo através de uma gestão financeira adequada, e com princípios corretos, deixando de encarar as finanças como tabus ou como algo intangível.

Para se ter um maior controle sobre o dinheiro e uma melhor eficiência no uso da receita, a gestão financeira é primordial. A gestão financeira pessoal pode ser entendida como a decisão de desenvolver uma estratégia para manter ou acumular bens e valores para formar um patrimônio pessoal ou familiar (Halfeld, 2006). Estratégia esta que pode ser para curto, médio ou longo prazo com foco na estabilidade econômica e financeira. No entanto, para que ocorra esse beneficiamento pessoal, é necessário ter uma apropriada alfabetização financeira.

De acordo com Vitt e colaboradores (2004) a alfabetização financeira desempenha um papel vital no processo de tomada de decisão financeira responsável, pois representa um esforço sistemático para desenvolver conhecimentos, comportamentos e atitudes financeiras positivas, uma vez que pode ser conceituada como "grau em que um indivíduo [...] possui a habilidade e confiança para administrar [...] suas finanças pessoais, por meio de decisões de curto prazo e planejamento financeiro de longo prazo, em meio aos eventos que ocorrem em sua vida [...]" (Remund, 2010, p. 284), como por exemplo, a vivência em meio a uma pandemia e uma crise mundial inesperada. Sendo que esta última, "influencia o movimento de estímulo e contenção das economias mundiais e tem reflexos nos padrões de consumo, podendo, em situações de oferta indiscriminada de crédito, favorecer o superendividamento das famílias" (Saleh \& Saleh, 2013).

Portanto, a educação financeira é considerada essencial para os indivíduos que estão à mercê de situações financeiras cada vez mais complexas (Potrich \&Vieiran \& Kirch, 2016). Diante do exposto, objetivou-se com essa pesquisa investigar o impacto econômico no início da pandemia do coronavírus COVID-19 sobre a família brasileira. 


\section{Metodologia}

A pesquisa foi realizada no mês de abril de 2020 e a partir de seu objetivo, caracteriza-se como quantitativa, em que uma amostra representativa assegura a possibilidade de generalização dos resultados (Günther, 2006), bem como qualitativa descritiva e/ou exploratória. Segundo Gil (2002, p. 41-57), a pesquisa é classificada em exploratória, descritiva e explicativa, no entanto, pode ser mais de um tipo, dependendo da sua concepção e dos seus propósitos. Em alguns momentos a pesquisa proposta tem como objetivo proporcionar maior familiaridade com o problema, se caracterizando como exploratória (Gil, 2002, p. 41) e em outros, apresenta características descritivas, que descreve determinada população ou fenômeno e ou estabelece relações entre variáveis (Gil, 2002, p. 42).

Além disso, possui um delineamento de pesquisa de campo, utilizando como instrumento de coleta de dados um questionário pré-estruturado com 19 perguntas objetivas, divididas nas duas categorias: (a) perfil ou dados pessoais (06 perguntas); e (b) o impacto financeiro causado pela pandemia do COVID-19 (13 perguntas). A aplicação do questionário foi aleatória, e por se tratar de um questionário on-line, foi utilizado para envio o e-mail e as redes sociais. O período para o recebimento das respostas foi estipulado em 15 dias (abril de 2020) e optou-se por não assentar um público alvo para a pesquisa, podendo assim, ter uma amostragem com um perfil representativo dos brasileiros.

No início do questionário os participantes precisavam concordar com o Termo de Consentimento Livre e Esclarecido (TCLE) para ter acesso às perguntas e participarem da pesquisa, afirmando seu interesse em participar do estudo de forma voluntária. Somente foram consideradas as respostas dos questionários que concordaram com o TCLE. A amostra foi composta por 339 respondentes, sendo destes, três $(n=03)$ não concordando com as condições impostas no TCLE para a participação da pesquisa, sendo desconsiderados no momento das análises. Por fim, a amostragem utilizada nas etapas posteriores de investigação apresentou um $n=336$ respondentes.

Os resultados foram extraídos do questionário on-line, tabulados e a indicação da frequência demonstra a quantidade de respondentes para cada opção de resposta, assim como, o percentual em relação a somatória da frequência para definir os grupos de respondentes. Deve-se destacar que algumas perguntas possuíam a opção de marcar mais de uma resposta e apresentar um somatório percentual maior que $100 \%$.

Neste estudo, utilizou-se o método de escores para definir a tendência de endividamento dos indivíduos após o início da pandemia do COVID-19. Segundo Pinheiro e Abrantes (2012), "por meio de escore é possível agrupar e classificar os indivíduos em segmentos semelhantes". Nesta pesquisa, o escore foi criado a partir dos resultados extraídos do questionário, descrito anteriormente, no qual as respostas que indicavam tendências ao endividamento foram tratadas como variáveis ordinais e receberam os valores de 0 a 3. Zero ponto para as respostas que não indicavam nenhum risco de endividamento, aumentando de forma crescente, até chegar em três pontos que correspondiam ao elevado risco de endividamento. O maior somatório de escores obtidos foi vinte (20).

\section{Resultados e Discussão}

O sistema financeiro, em 2020, teve sua estabilidade ameaçada pela crise instituída durante a pandemia de COVID-19 e "[...] quando se fala da análise de crises financeiras que foram originadas em determinado mercado e transmitidas rapidamente para mercados de outras regiões ou países, há um interesse dos pesquisadores pelo olhar do contágio financeiro" (Barros et al., 2020). Frente a isso, a pesquisa foi realizada com o intuito de investigar o impacto econômico da crise causada pela pandemia do COVID-19 sobre a família brasileira. Para isso, foram entrevistados 339 indivíduos, sendo estes de 17 estados brasileiros, através de um questionário on-line. Do total, $99,1 \%(n=336)$ dos sujeitos atenderam ao convite para 
participar da pesquisa. Ainda em nosso estudo, 0,9\% $(n=3)$ não concordaram com o Termo de Consentimento Livre e Esclarecido (TCLE), sendo desconsiderado nas análises dos dados.

A média de idade dos entrevistados foi de 35 anos e oito meses, sendo a menor e a maior idade de 16 e 68 anos, respectivamente. Obteve-se um maior número de respondentes do sexo feminino, com 65, 2\% (n=219), contra 34,5\% (n=116) do sexo masculino. Rio Grande do Sul, Minas Gerais e São Paulo, dentre os 17 estados que tiveram participantes, foram os que tiveram o maior número de respondentes com 51,2\% (n=172), 18,5\% (n=62) e 14,9\% (n=50), respectivamente. Entretanto, os estados do Amapá, Distrito Federal e Rio Grande do Norte foram os de menor expressão com 0,3\% (n=1).

Quando questionado sobre o número de pessoas residentes em casa, 31,0\% ( $\mathrm{n}=104)$ afirmam morar um total de três, 26,2\% (n=88) duas e 0,3\% (n=1) apenas uma pessoa. Outro estudo relatou que a média de pessoas por residência durante esse período pandêmico foi de 3,2, sendo que, o alto percentual vivendo em residências com 2 a 4 pessoas (Bezerra et al., 2020). Em relação a renda familiar, observou-se uma família $(0,3 \%)$ com renda inferior a $\mathrm{R} \$ 500,00$ e cinquenta e oito famílias $(17,7 \%)$ superior a R $\$ 9.405,00$ (maior que 9 salários mínimos). No entanto, o maior percentual de famílias possui renda mensal entre R\$1.045,00 e R\$3.135,00 (1 a 3 salários mínimos).

Em um estudo realizado por Bezerra e colaboradores (2020) foi levantado que entre as pessoas com maior grau de instrução, ou seja, com mais estudo e maiores rendas o aspecto mais afetado foi o convívio social. Já para o perfil populacional inverso, baixa renda e escolaridade, a preocupação está relacionada com questões financeiras. Os autores supracitados também destacam que a percepção dos entrevistados em relação ao isolamento social como medida de prevenção da pandemia teve variação de acordo com a renda, a escolaridade, idade e sexo. No entanto, em sua maioria, acreditam que a medida é essencial e estão dispostos a contribuir com enfrentamento à COVID-19.

Devido ao impacto econômico nas famílias gerado pela crise, proveniente da pandemia, um grande número de pessoas perdeu ou teve sua jornada de trabalho reduzida e consequentemente, diminuição dos seus recursos financeiros. Nos dados apresentados por Opinion Box (2020), 22\% dos entrevistados relataram estar sem trabalhar, no contexto da pandemia, crescendo para 26\%. Além disso, em um estudo realizado pelo Sebrae (2020), para avaliar o impacto da pandemia do coronavírus nas pequenas empresas, mostra que entre as micro e pequenas empresas, 34,2\% relataram perda total de seus negócios e não realizaram nenhuma atividade econômica, aumentando para 52,2\% nos microempreendedores individuais, ficando assim impossibilitados de realizar suas operações. Diante disso, fez-se necessário a busca por atividades alternativas remuneradas, ou seja, uma segunda profissão. Ao encontro disso, 17,0\% (n=57) dos entrevistados disseram ser necessário desenvolver uma segunda função gratificada para somar com as receitas e auxiliar nas despesas mensais familiares. Além disso, 32,1\% (n=108) avaliam que será necessário em algum momento da pandemia buscar uma atividade extra para complementar a renda. A necessidade da busca por uma profissão alternativa pode estar vinculada a dois fatores: a duração da pandemia e a diminuição ou finalização das reservas financeiras.

O momento financeiro crítico atual é uma situação nova para a maioria, se não, para todas as famílias brasileiras. Visando verificar o preparo das mesmas em relação às situações emergenciais pontuais, como essa de pandemia, foram questionadas o preparo financeiro para o enfrentamento deste período minucioso global e 43,5\% ( $\mathrm{n}=146)$ dos respondentes julgaram não estar preparados. Devemos ressaltar que 46,1\% $(n=155)$ afirmaram ter uma reserva financeira para períodos emergenciais, contradizendo o resultado anterior. Além disso, 31,0\% ( $\mathrm{n}=104)$ e 25,5\% ( $\mathrm{n}=86)$ estavam parcialmente e totalmente preparados financeiramente para o enfrentamento da pandemia, respectivamente.

Com a crise, e consequentemente, redução das receitas mensais houve a necessidade de realizar cortes de gastos para que pudesse ser mantida a saúde financeira familiar. Do total de entrevistados, $50,3 \%(n=169)$ relataram que a renda mensal foi reduzida, sendo destes $23,8 \%(n=80)$ com redução de até $25 \%$ de suas finanças; já os que tiveram entre 25-50\% e mais de 50\% 
de corte representam 14,0\% $(n=47)$ e 12,5\% $(n=42)$, respectivamente. Até o momento do levantamento dos dados, uma alta porcentagem $(49,7 \%$ - $n=177)$ dos entrevistados não relataram cortes em suas receitas mensais.

Diante disso, 56\% $(\mathrm{n}=188)$ dos sujeitos relataram estar sendo necessário realizar cortes para manter o controle das finanças. Dentre as despesas básicas do dia a dia, as pessoais e de lazer, foram ranqueadas como sendo prioridade nos cortes. Em contrapartida, 30,1\% julgam não ser oportuno realizar nenhum tipo de corte, seja por não ter a renda mensal familiar alterada $(24,1 \%$ - $n=81)$ ou por possuir algum tipo de reserva financeira $(6 \%$ - $n=20)$. Cabe ressaltar, que parte dos entrevistados $(14 \%$ - n=47) estão em alerta quanto aos cortes familiares caso a pandemia se estenda por um maior período. As despesas priorizadas no período de pandemia serão alimentação e saúde.

O impacto do isolamento sobre a questão social na renda/gastos das pessoas foi investigado por Bezerra e colaboradores (2020) e, os mesmos, chegaram à conclusão de que em 32\% dos entrevistados não está tendo impacto na renda/gastos; 34\% iniciaram alguma economia de dinheiro; 13\% aumentaram os gastos; e 20\% perderam a sua renda em função do isolamento social.

Com apenas dois meses de início do isolamento no Brasil, um percentual de 13,7 (n=46) dos entrevistados já haviam contraído dívidas pelas necessidades que surgiram decorrentes da pandemia. Levando em consideração os prazos, sendo estes, curto e médio, para quitação de suas obrigações, 8,9\% $(n=30)$ e 4,8\% $(n=16)$ se enquadram nestas categorias, respectivamente. Em contrapartida, 86,3\% não tinham angariado nenhum tipo de despesa, sendo que destes, 11,0\% (n=37) acreditam assumir alguma dívida a qualquer momento caso a crise se estenda por mais tempo. Diante disso, para evitar o acúmulo de contas uma parcela dos respondentes $(24,7 \%$ - n=83) afirmaram que foi necessário o uso de suas reservas durante esse período pandêmico. Alguns, 16,1\% (n=54) acreditam que futuramente utilizarão suas economias e 36,6\% (n=123) ainda não têm uma definição certa em relação ao questionamento. Somente $22,6 \%(n=76)$ da população amostral da pesquisa não precisarão se desfazer de seus rendimentos.

Uma parcela reduzida da população amostral da pesquisa, 17,8\% (n=60), começou a utilizar cartão de crédito, talão de cheque e limite do cheque especial, sendo: 8,0\% $(n=27) ; 0,9 \%(n=3)$; e 8,9\% $(n=30)$, respectivamente. A maior parte, $83,3 \%$ $(\mathrm{n}=280)$, não precisou acessar nenhuma das categorias de crédito supracitadas ou já utilizavam as mesmas anteriormente a pandemia. Em adição, 1,2\% (n=4), marcaram mais de uma opção, sendo conjuntamente, a aquisição de um cartão de crédito e utilização do limite do cheque especial $(n=3)$ e a aquisição de um cartão de crédito, mas, que ainda não foi necessário utilizálo. A situação global e local, associada ao contínuo desenvolvimento tecnológico e sistemas de crédito, favorece o aumento do consumo, enquanto a falta de educação financeira favorece o endividamento (Brasil, 2010).

A falta de controle dos gastos e a redução salarial oriundo da crise financeira induz, muitas vezes, os indivíduos a entrarem em uma situação emergencial. Medidas pessoais devem ser tomadas para a quitação de dívidas, como por exemplo, a venda de bens financiados para redução dos gastos fixos mensais, tal qual desfazer de bens liquidados. Considerando a amostragem total, apenas $1,8 \%(n=6)$ avaliaram ter que desfazer de algum ativo imobilizado o mais rápido possível e 21,1\% $(n=71)$ talvez necessite caso a pandemia tenha uma extensão de um longo período. Aqueles que afirmaram não ser necessário desfazer dos seus bens foram $77,1 \%(n=259)$ dos respondentes.

Durante esse momento de pandemia, os indivíduos foram obrigados a intensificar ou iniciar o hábito de comprar de forma on-line, uma vez que, o isolamento obrigatório impediu os mesmos de estarem se deslocando aos estabelecimentos para a realização da compra. Em tal caso, 31,5\% (n=106) aumentaram a aquisição de produtos pela internet e em adição, 8,0\% $(n=27)$, deram início a essa modalidade de compra. Devemos ressaltar que 34,2\% (n=115) ainda não realizaram compras em comércio eletrônico, mas, pretendem em breve e 26,2\% (n=88) afirmaram ser adeptos às compras presenciais.

Para verificar o aprendizado dos respondentes em relação às dificuldades enfrentadas por esse longo período de pandemia e da grave crise financeira, foi perguntado: “Após a pandemia, você iniciará uma reserva financeira para possíveis 
emergências?". Mesmo passando por um extensivo momento emergencial, 4,8\% $(\mathrm{n}=16)$ dos entrevistados ainda não pretendem poupar recursos para eventuais contingências. Além desses, 24,1\% (n=81), ainda possuem dúvidas em relação a economizar recursos com a finalidade de suprir futuros contratempos. Ditosamente, uma parcela considerada, 71,1\% (n=239), perceberam a importância de se realizar uma reserva para este tipo de situação, mostrando a necessidade das famílias brasileiras terem maior consciência e incentivo na busca por uma educação financeira.

Com o enfrentamento de uma crise financeira séria, o governo brasileiro publicou no Diário Oficial da União no dia 02 de abril de 2020 a lei no 13.982 que dispõe sobre:

“[...] parâmetros adicionais de caracterização da situação de vulnerabilidade social para fins de elegibilidade ao benefício de prestação continuada (BPC), e estabelece medidas excepcionais de proteção social a serem adotadas durante o período de enfrentamento da emergência de saúde pública de importância internacional decorrente do coronavírus (Covid-19) responsável pelo surto de 2019, a que se refere a Lei n 13.979, de 6 de fevereiro de 2020”.

Com isso, buscamos verificar entre os entrevistados aqueles que receberiam o benefício concedido pelo governo no valor de $\mathrm{R} \$ 600,00$. Dos 336 respondentes, 12,5\% (n=42) afirmaram fazer parte dos beneficiários, contra 87,5\% (n=294) que não seriam contemplados.

A tendência de endividamento dos entrevistados foi analisada utilizando escores, no qual variou entre 0 e 20 escores (Figura 1). Sendo 0 nenhum e 20 escores indicando alto risco de endividamento.

Figura 1 - Tendência de endividamento durante o período de pandemia

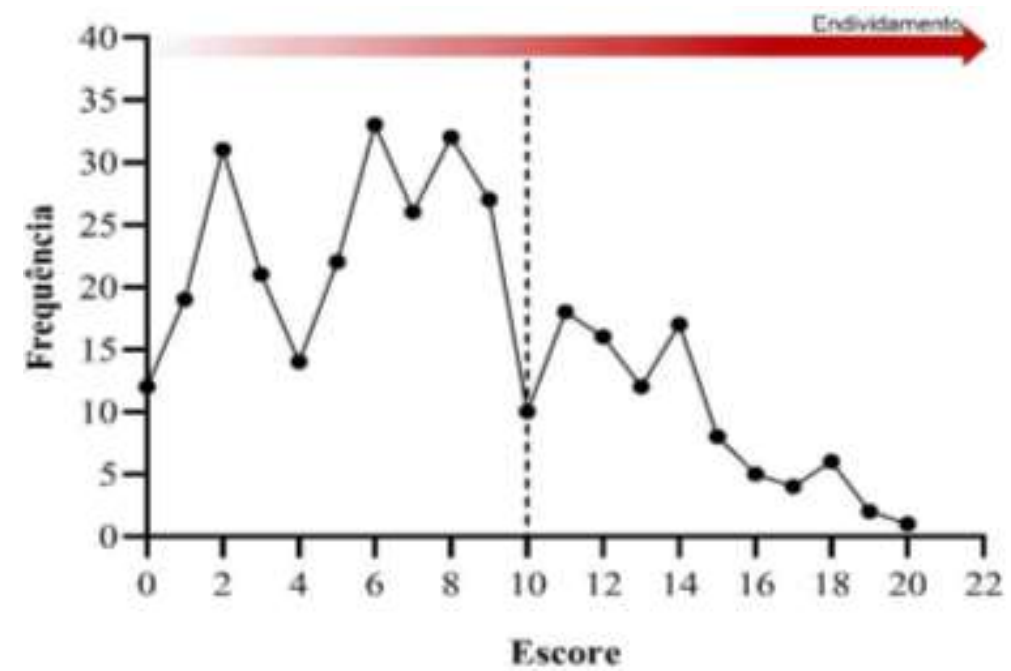

Fonte: Autores (2021).

Considerando, o escore médio igual a 10, observamos que 73,5\% (n=247) dos respondentes estão abaixo desse valor mediano (grupo A), em contrapartida, 26,5\% (n=89) apresentaram valores de escores acima da média (grupo B). Devemos chamar atenção, que o grupo B apresentou uma queda gradual na frequência com o aumento crescente do escore.

Considerando um total de 336 respondentes, 12 não correm nenhum risco de endividamento apresentando escore 0 e 1 respondente tende a se endividar apresentando o escore máximo que é 20. Dez indivíduos estão em uma zona mediana com escores igual a 10 . 


\section{Conclusão}

Com o objetivo de investigar o impacto econômico no início da pandemia do coronavírus COVID-19 sobre a família brasileira, os resultados apresentados neste estudo podem sustentar pelo menos três considerações: as famílias brasileiras não estão preparadas financeiramente para o enfrentamento de situações emergenciais como de uma pandemia; mesmo diante de preocupações oriundas de endividamento, causado pela pandemia, não é percebido uma motivação para economizar recursos para eventuais contingências emergenciais; e que o impacto financeiro causado pela pandemia nas famílias brasileiras é intensificado pelo analfabetismo financeiro. Mesmo no início da pandemia já é perceptível um impacto negativo da crise mundial nas famílias, com redução de jornadas de trabalho, assim acarretando diminuição nos proventos mensais, procura de complementação de renda através de uma segunda atividade remunerada, cortes de despesas pessoais e atividades de lazer e utilização das reservas financeiras. O controle das finanças é indispensável para a saúde financeira de uma família, uma vez que, induz o pensamento de lidar com receitas e despesas e de preparar para condições adversas. Entende-se que a educação financeira pode melhorar e/ou ajudar as famílias no enfrentamento de situações emergenciais, não somente na área da saúde como em uma pandemia, como também no âmbito social em momentos de crises mundiais.

A pesquisa foi realizada no início do período pandêmico e os impactos gerados pela crise mundial ainda eram brandos. Diante disso, é necessário a realização de novas pesquisas avaliando o impacto da pandemia sobre a população brasileira nos dias atuais. Além do que, pesquisas voltadas para a educação financeira também são necessárias, visando não somente avaliar a saúde financeira da população, como também investigar novas metodologias de transmissão de conhecimento sobre finanças que consigam atingir todos os níveis sociais dos brasileiros. A temática educação financeira versus crises financeiras devem ser o foco de trabalhos futuros, principalmente no ensino básico de educação.

\section{Referências}

Amadeu, J. R. (2009). A educação financeira e sua influência nas decisões de consumo e investimento: proposta de inserção da disciplina na matriz curricular.

Baldwin, R. (2020). To treat COVID-19's economic impact, start by keeping the lights on. Chicago Booth Review. https://review. chicagobooth. edu/economics/2020/article/treat-covid-19-s-economic-impact-startkeeping-lights.

Behrens, M. A. (2010) Docência Universitária num Paradigma da Complexidade: possibilidades de formação continuada no strictu sensu. In: Revista Diálogo Educacional, 10(29), 27-44.

Bezerra, A. C. V., Silva, C. E. M. D., Soares, F. R. G., \& Silva, J. A. M. D. (2020). Fatores associados ao comportamento da população durante o isolamento social na pandemia de COVID-19. Ciência \& Saúde Coletiva, 25, 2411-2421.

Brasil. (2000). Ministério da Educação. Secretaria de Educação Média e Tecnológica. Parâmetros Curriculares Nacionais: Ensino Médio. Brasília: Ministério da Educação.

Brasil. (2010). Presidência da República. Casa Civil. Subchefia para assuntos jurídicos. Decreto n 7.397 , de 22 de dezembro de 2010 . Diário Oficial da União, Poder Executivo, Brasília, DF, 23 dez. 2010. Seção 1, Edição Extra, p.7.

Brasil. (2020). Lei Complementar $\mathrm{n}^{\circ}$ 13.982, de 02 de abril de 2020. Estabelece medidas excepcionais de proteção social a serem adotadas durante o período de enfrentamento da emergência de saúde pública de importância internacional decorrente do coronavírus (Covid-19) responsável pelo surto de 2019. Brasília.

Brasil. (2020). Ministério da Saúde. Coronavírus (COVID-19). Available at: https://www.gov.br/saude/pt-br/vacinacao/\#o-que-e-covid

Cordeiro, N. J. N., Costa, M. G. V., \& da Silva, M. N. (2018). Educação Financeira no Brasil: uma perspectiva panorâmica. Ensino da Matemática em Debate, $5(1), 69-84$

Forbes, K., \& Rigobon, R. (2001). Measuring contagion: conceptual and empirical issues. In International financial contagion (pp. 43-66). Springer, Boston, MA.

Gil, A. C. (2002). Como elaborar projetos de pesquisa. 4. ed. São Paulo: [s. n.].

Günther, H. (2006). Pesquisa qualitativa versus pesquisa quantitativa: esta é a questão? Psicologia: teoria e pesquisa, 22(2), 201-209.

Kruger, F. (2014). Avaliação da educação financeira no orçamento familiar. Trabalho de conclusão de curso (TCC). Fundação Adolpho Bósio de Educação no Transporte (FABET). Faculdade de Tecnologia Pedro Rogério Garcia (FATTEP) Santa Catarina. 
Research, Society and Development, v. 10, n. 11, e55101119389, 2021

(CC BY 4.0) | ISSN 2525-3409 | DOI: http://dx.doi.org/10.33448/rsd-v10i11.19389

Matta, R. O. B. (2007). Oferta e demanda de informação financeira pessoal: o Programa de Educação Financeira do Banco Central do Brasil e os universitários do Distrito Federal. Dissertação de mestrado, Universidade de Brasília, Brasília, DF, Brasil.

Mulligan, C. B., \& Murphy, K. M., \& Topel, R. H. (2020). Some basic economics of COVID-19 policy. Chicago Booth Review, April, 27.

OECD, \& Development Staff (2005). Improving Financial Literacy: Analysis of Issues and Policies. Organisation for Economic Co-operation and Development.

Opinion Box. (2020). Impacto nos hábitos de compra e consumo: como a pandemia está transformando o comportamento do consumidor brasileiro. Nota Técnica 2 ed. Belo Horizonte. https://blog.opinionbox.com/dados-atualizados-sobre-o-coronavirus/

Pinheiro, M. C. D. O., \& Abrantes, S. D. M. P. (2012). Avaliação da Exposição aos Corantes Artificiais presentes em balas e chicletes por crianças entre 3 e 9 anos estudantes de escolas particulares da Tijuca/Rio de Janeiro. Analytica (online), 10(58), 02-12.

Potrich, A. C. G., \& Vieira, K. M., \& Kirch, G. (2016). Você é alfabetizado financeiramente? Descubra no termômetro de alfabetização financeira. Base Revista de Administração e Contabilidade da UNISINOS, 13(2), 153-170.

Remund, D. L. (2010). Financial literacy explicated: the case for a clearer definition in an increasingly complex economy. Journal of Consumer Affairs, 44(2), 276-295.

Ross, S. A., Westerfield, R. W., Jordan, B. D., \& Lamb, R. (2013). Fundamentos de administração financeira. AMGH Editora.

Saleh, A. M., \& Saleh, P. B. D. O. (2013). O elemento financeiro e a Educação para o Consumo Responsável. Educação em Revista, 29 (4), 189-214.

Ás Micro, S. B. D. A., \& Empresas, P. (2020). O impacto da pandemia do coronavírus nos pequenos negócios - 3 a edição. Coleta: 30 de abril a 5 de maio de 2020. https://datasebrae.com.br/wp-content/uploads/2020/05/Impacto-do-coronav\%C3\%ADrus-nas-MPE-3\%C2\%AAedicao_Mei-MPE-e-geral.pdf

Seth, N., \& Panda, L. (2018). Financial contagion: review of empirical literature. Qualitative Research in Financial Markets, 10(1), 15-70.

Scott, J. (2020). The economic, geopolitical and health consequences of COVID-19. In World Economic Forum. World Economic Forum.

University \& Medicine John Hopkins (2021). Coronavirus Resource Center. https://coronavirus.jhu.edu/map.html.

Verdélio, A. (2020). Pandemia leva governo a adotar medidas econômicas e de atenção à saúde. https://agenciabrasil.ebc.com.br/saude/noticia/202003/emergencia-em-saude-e-estado-de-calamidade-veja-diferenca.

Vitt, L. A. (2004). Consumers' financial decisions and the psychology of values. Journal of Financial Services Professionals, 68-77. 